\title{
Dengue Virus Infections among Peace Corps Volunteers in Timor-Leste, 2018-2019
}

Liliana Sánchez-González, ${ }^{1 *}$ Margaret Venuto, ${ }^{2}$ Scott Poe, ${ }^{2}$ Chelsea G. Major, ${ }^{1}$ Leonardus Baskara, ${ }^{3}$ Sevinj Abdiyeva, ${ }^{3}$ Daniel Murphy, ${ }^{2}$ Jorge L. Munoz-Jordan, ${ }^{1}$ Freddy A. Medina, ${ }^{1}$ Gabriela Paz-Bailey, ${ }^{1}$ Kyle Petersen, ${ }^{2}$ Karen Becker, ${ }^{2}$ and Tyler M. Sharp ${ }^{1,4}$

${ }^{1}$ Dengue Branch, Centers for Disease Control and Prevention, San Juan, Puerto Rico; ${ }^{2}$ Epidemiology and Surveillance Unit, Office of Health Services, United States Peace Corps, Washington, District of Columbia; ${ }^{3}$ Timor-Leste Country Office, Office of Health Services, U.S. Peace Corps, Washington, District of Columbia; ${ }^{4}$ U.S. Public Health Service, Rockville, Maryland

\begin{abstract}
Dengue is an ongoing health risk for Peace Corps Volunteers (PCVs) working in the tropics. On May 2019 the Peace Corps Office of Health Services notified the Centers for Disease Control and Prevention (CDC) of a dengue outbreak among PCVs in Timor-Leste. The purpose of this investigation was to identify the clinical, demographic, and epidemiological characteristics of PCVs with dengue and recommend dengue preventive measures. To identify PCVs with dengue and describe disease severity, the medical records of PCVs reporting fever during September 2018-June 2019 were reviewed. To identify factors associated with dengue virus (DENV) infection, we administered a questionnaire on demographics, travel history, and mosquito avoidance behaviors and collected blood specimens to detect the antiDENV IgM antibody to diagnose recent infection. Of $35 \mathrm{PCVs}$ in-country, 11 (31\%) tested positive for dengue (NS1, IgM, PCR), eight requiring hospitalization and medical evacuation. Among 27 (77\%) PCVs who participated in the investigation, all reported having been recently bitten by mosquitoes and $56 \%$ reported being bitten most often at home; only $16(59 \%)$ reported having screens on bedroom windows. Nearly all (93\%) PCVs reported using a bed net every night; fewer $(70 \%)$ reported using mosquito repellent at least once a day. No behaviors were significantly associated with DENV infection. Raising awareness of dengue risk among PCVs and continuing to encourage mosquito avoidance behavior to prevent dengue is critical. Access to and use of measures to avoid mosquito bites should be improved or implemented. Peace Corps medical officers should continue to receive an annual refresher training on dengue clinical management.
\end{abstract}

\section{BACKGROUND}

Dengue is the most common arboviral disease globally and remains a risk for both residents of and travelers to tropical and subtropical regions. ${ }^{1}$ Most dengue virus (DENV) infections are asymptomatic, ${ }^{2}$ and common signs and symptoms among symptomatic patients include fever, myalgia, arthralgia, rash, and minor hemorrhagic manifestations (e.g., petechiae, gingival bleeding). ${ }^{3}$ Diagnosis of dengue is complicated by the overlap of its clinical manifestations with those of other acute febrile illnesses including other arboviral diseases (e.g., chikungunya, Zika) and by limited access to laboratory testing. Roughly $5 \%$ of patients may progress to severe dengue, which is characterized by increased vascular permeability leading to shock, hemorrhage, and, in some cases, death. ${ }^{3}$ Severe dengue is often preceded by warning signs (e.g., abdominal pain, lethargy, and hepatomegaly). ${ }^{4,5}$ Early recognition of warning signs and appropriate clinical management are key to improving clinical outcomes among dengue patients and have been shown to reduce the case-fatality rate among dengue patients from $\approx 5 \%$ to $<0.5 \%$. ${ }^{6}$

Dengue is endemic in Timor-Leste where mosquito vectors Aedes aegypti and Aedes albopictus are present, and multiple epidemics have occurred over the past two decades. ${ }^{7,8} \mathrm{Be}-$ cause of poor access to and use of health care, high casefatality rates (i.e., $12 \%$ ) have been reported during prior epidemics. ${ }^{9,10}$ During January-April 2019, the World Health Organization (WHO) office in Timor-Leste reported an increase in the number of cases of dengue $(n=532)$ compared with the same period in the previous year $(n=317)^{11}$ and 5 deaths, consistent with a dengue epidemic. ${ }^{7}$

*Address correspondence to Liliana Sánchez-González, 1324 Calle Cañada, San Juan, Puerto Rico 00920. E-mail: naq5@cdc.gov
The Peace Corps, an independent U.S. government agency, assigns volunteers (PCVs) to 64 developing countries to work on different projects including education, health, agriculture, and environment. PCVs typically serve 27 months at posts in rural areas with limited infrastructure and increased risk of exposure to various communicable diseases. Because they usually live with local families in areas that are often isolated and lack vector control programs, the risk of dengue among PCVs is high. Among dengue endemic countries where PCVs were serving between 2000 and 2014, incidence of dengue was highest for those in Timor-Leste (11 cases per 1,000 volunteer-months). ${ }^{12}$

On May 6, 2019, CDC was notified by the Peace Corps Office of Health Services of a recent increase in dengue cases among PCVs in Timor-Leste. Because of the ongoing dengue epidemic exacerbated by limited access to adequate medical care in-country, the Peace Corps requested assistance from CDC to identify and gain a better understanding of factors associated with DENV exposure among PCVs and to provide recommendations to mitigate the risk of exposure among PCVs serving in Timor-Leste and the community.

The objectives of this investigation were to: 1) describe the clinical characteristics and outcomes among PCVs with febrile illness during the dengue outbreak in Timor-Leste in 2018-2019; 2) identify clinical or demographic characteristics associated with PCVs with dengue; 3) identify risk factors associated with infection with DENV or other arboviruses; and 4) recommend prevention and control measures to protect PCVs serving in Timor-Leste from arboviral infection.

\section{METHODS}

Participant recruitment and data collection. The investigation consisted of two parts: 1) Review of medical records for PCVs who had sought medical care for fever or suspicion of dengue during September 2018-June 2019 
(referred to as "medical records review") and 2) A survey and blood collection for additional testing (referred to as "survey and blood sample collection").

First, for the medical records review, for any PCVs in TimorLeste who sought medical care for an acute febrile illness or dengue suspicion during September 2018-June 2019, clinical data and DENV laboratory results were abstracted from medical records in the Peace Corps Medical Electronic Documentation and Inventory Control System (PCMEDICS). These data were evaluated using the WHO 2009 dengue case definitions. $^{1}$

For the second part, the survey and blood sample collection, with the support of Peace Corps medical officers, PCVs in-country at the time of these activities $(n=35)$ were provided an overview on the purpose and procedures of this part of the investigation and invited to participate. Questionnaires were completed and data samples collected between July 13th and July 19th, 2019. PCVs who agreed to participate: 1) completed a one-time self-administered questionnaire on demographics, recent illness history, vaccinations, clinical care, and mosquitobite prevention behaviors during the previous six months, October 2018-June 2019, and 2) provided a blood specimen for diagnostic testing to detect recent infection with DENV, chikungunya virus (CHIKV), and Zika virus (ZIKV). All PCVs were serving in Timor-Leste for the complete period of interest for this investigation. Diagnostic testing was performed at the request of the medical officers, who returned test results to PCVs.

Definitions. For the medical record review, we defined "dengue patients" as PCVs who sought medical care for acute febrile illness or other acute symptoms and had laboratory diagnostic evidence of DENV infection (i.e., positive result for DENV NS1, anti-DENV IgM, or DENV RT-PCR).

For the survey and blood sample collection, we defined "participants" as PCVs in Timor-Leste at the time of the investigation who answered the questionnaire and provided a blood sample. Participants with previously documented DENV infection through the medical records review and/or through diagnostic testing performed at the CDC Dengue Branch are included in the DENV infection group for the survey and blood sample collection analysis.

Diagnostic testing. For PCVs identified through the medical records review who didn't participate in the survey and blood sample collection, no additional testing was performed. Serum specimens of the participants of the survey and blood sample collection were stored at $-20^{\circ} \mathrm{C}$ until being shipped on dry ice to the CDC Dengue Branch in San Juan, Puerto Rico, where they were stored at $-70^{\circ} \mathrm{C}$. Specimens were tested by IgM ELISA to detect evidence of recent infection with DENV, ${ }^{13}$ ZIKV, ${ }^{14}$ or CHIKV. ${ }^{15}$ Specimens positive for anti-ZIKV and anti-CHIKV IgM antibody by ELISA were tested by plaque reduction neutralization assay (PRNT). Testing plan included PRNT for any positive anti-DENV specimen from participants without a previous viral test for confirmation. RT-PCR to detect DENV, CHIKV, and ZIKV nucleic acid was not performed because all participants were asymptomatic at the time of specimen collection.

Data analysis. For the medical record review, we used medical record data to compare demographic and clinical characteristics of dengue patients to those people who sought care for an acute febrile illness without laboratory diagnostic evidence of dengue. For the survey and blood sample collection, we used questionnaire responses to compare participants with and without laboratory diagnostic evidence of DENV infection to identify risk factors associated with DENV infection among PCVs. Pearson's $x^{2}$ test and Fisher's exact test were used to compare categorical variables between groups. Median 2-sample test was used to compare continuous variables. For all tests, $P$ values less than 0.05 were considered statistically significant. Data analyses were performed using SAS 9.4 (Cary, NC) statistical software.

Human subjects. CDC's Human Subjects Office reviewed this investigation's protocol and provided a nonresearch determination because it was considered part of a public health response. Participation was voluntary, and responses to survey questions were confidential. Identifiable data from questionnaires was not shared with Peace Corps medical officers or Headquarters staff. All participants were guaranteed that their answers, including compliance with dengue prevention measures, would not have any negative impact. All participants provided written consent. PCVs provided permission to the Peace Corps to access medical records.

The presentation and discussion of findings follow the Strengthening the Reporting of Observational Studies in Epidemiology (STROBE) reporting guidelines.

\section{RESULTS}

Medical records review-Clinical characteristics and
outcomes among PCVs with febrile illness or suspicion of dengue $(n=20)$. PCVs typically receive their medical care from PC medical officers first, who then refer them to local hospitals when needed. Through review of medical records in PCMEDICS, 20 PCVs were identified who had sought medical care through their PC Medical Office for acute febrile illness or suspicion of dengue during September 2018-June 2019 among the 35 PCVs in-country. Dengue diagnostic testing had been performed for all during their medical care using the Dengue Duo (Standard Diagnostics, Inc.) test, which detects DENV NS1 antigen and anti-DENV IgM antibody. Five (25\%) PCVs were also tested by RT-PCR to detect DENV nucleic acid.

In total, 11 (55\%) of the 20 PCVs with acute febrile illness or suspicion of dengue had laboratory evidence of DENV infection. Laboratory tests for DENV diagnosis were conducted according to days post-onset (DPO) and availability in local and referral hospitals. Typically, patients managed in TimorLeste were tested by the rapid NS1/lgM assay, whereas those people who were med-evacuated to Thailand were tested by RT-PCR. Median of DPO testing was 1.5 days (range 09 days). Testing for dengue NS1, IgM, and IgG was conducted among all 20 PCVs. NS1 was detected among 10 PCVs (Median DPO = 1, range 0-6), of which four were also positive for anti-DENV IgM antibody (Median DPO = 2.5, range 0-6). Five PCVs were positive for DENV RNA by RT-PCR (Median $\mathrm{DPO}=1$, range 1-3): one was negative for NS1, three were positive for NS1, and one was positive for NS1 and anti-DENV IgM antibody. Among PCVs that tested positive by RT-PCR, DENV-1 $(n=2)$, and DENV-3 $(n=3)$, serotypes were detected.

We compared demographic and clinical characteristics of dengue patients to those who sought care for an acute febrile illness and had no laboratory diagnostic evidence of dengue (Table 1). Median age among all patients was 24 years (range: 22-25) and half (50\%) were female. Nausea, vomiting, and 
TABLE 1

Characteristics of Peace Corps volunteers in Timor-Leste with fever or clinical suspicion of dengue by status of dengue virus infection, September 2018-June $2019(n=20)$

\begin{tabular}{|c|c|c|c|c|}
\hline & $\begin{array}{l}\text { All patients with AFI or } \\
\text { dengue suspicion, } n=20 \text { (\%) }\end{array}$ & $\begin{array}{l}\text { Patients with AFI or dengue suspicion and evidence of } \\
\text { DENV infection, } n=11(\%)\end{array}$ & $\begin{array}{l}\text { Patients with AFI or dengue suspicion and no } \\
\text { evidence of DENV infection, } n=9(\%)\end{array}$ & $P$ value ${ }^{*}$ \\
\hline \multicolumn{5}{|l|}{ Demographic characteristics } \\
\hline Age in years, median (range) & $24(22-45)$ & $26(22-45)$ & $23(22-27)$ & 0.272 \\
\hline Female gender & $10(50)$ & $7(64)$ & $3(33)$ & 0.369 \\
\hline \multicolumn{5}{|l|}{ Signs and symptoms, $n(\%)$} \\
\hline Fever & $19(95)$ & $11(100)$ & $8(89)$ & 0.450 \\
\hline Nausea or vomiting & $14(70)$ & $8(73)$ & $6(67)$ & 1.000 \\
\hline Persistent vomiting & $3(15)$ & $2(18)$ & $1(11)$ & 1.000 \\
\hline Rash & $9(45)$ & $8(73)$ & $1(11)$ & 0.009 \\
\hline Headache & $13(65)$ & $8(73)$ & $5(56)$ & 0.642 \\
\hline Eye pain & $3(15)$ & $3(27)$ & $0(0)$ & 0.218 \\
\hline Arthralgia & $3(15)$ & $3(27)$ & $1(11)$ & 0.218 \\
\hline Myalgia & $17(85)$ & $11(100)$ & $6(67)$ & 0.073 \\
\hline Abdominal pain/tenderness & $3(15)$ & $1(9)$ & $2(22)$ & 0.565 \\
\hline Diarrhea & $5(25)$ & $1(9)$ & $4(44)$ & 0.127 \\
\hline Cold skin/cold extremities & $1(5)$ & $1(9)$ & $0(0)$ & 1.000 \\
\hline Mottled skin & $1(5)$ & $1(9)$ & $0(0)$ & 1.000 \\
\hline
\end{tabular}

headache were common among all patients, and all dengue patients presented with fever and myalgia. Rash was significantly more common in dengue patients $(73 \%$ versus $11 \%$, respectively; $P<0.05)$. No other signs and symptoms or demographic characteristics were significantly associated with dengue patients.

Dengue warning signs were reported in three dengue patients. One patient who tested positive for DENV-3 by RT-PCR had persistent vomiting and a pulse pressure $<20$. The other two patients, who tested positive for DENV by NS1, presented with bleeding (menorrhagia) and persistent vomiting, as well as abdominal pain and ascites, respectively. Clinical findings among dengue patients also included hypotension and marked thrombocytopenia, but none met the case definitions for severe dengue or dengue hemorrhagic fever.

The first patient with laboratory diagnostic evidence of DENV infection had symptom onset in January 2019. Additional cases were identified through June 2019 (Figure 1). The greatest numbers of monthly dengue patients were identified in February and March $(n=3)$.

Survey and blood sample collection-Factors associated with DENV infection $(\boldsymbol{n}=\mathbf{2 7})$. Among $35 \mathrm{PCV}$ s in Timor-
Leste at the time of the investigation, 27 (77\%) participated in the survey and blood sample collection aspect of this investigation. This includes 13 out of the 20 PCVs identified in the medical records review previously described (Figures 2 and 3). Among all participants, 17 (63\%) were male, and median age was 25 years (range: 22-67). Most participants reported having been vaccinated against Japanese encephalitis (JE) $(n=21,78 \%)$ and 7 (26\%) against yellow fever (YF). Seven (26\%) PCVs reported having lived in a dengue-endemic area before moving to Timor-Leste. Two (7\%) reported having been diagnosed with dengue prior to arriving in Timor-Leste.

Anti-DENV IgM antibody was detected in serum specimens from two (7\%) participants, both of whom had been diagnosed with dengue in 2019 and identified in the first section of this paper through the medical records review. As these participants had a positive viral test documented in their medical record, PRNT testing for DENV was not conducted. Anti-ZIKV IgM antibody was detected in one PCV for whom anti-DENV IgM antibody was not detected; confirmatory testing by PRNT did not detect neutralizing anti-ZIKV or -DENV antibodies. As the PCV reported having been recently vaccinated against JE, the positive anti-ZIKV IgM result may have been a result of

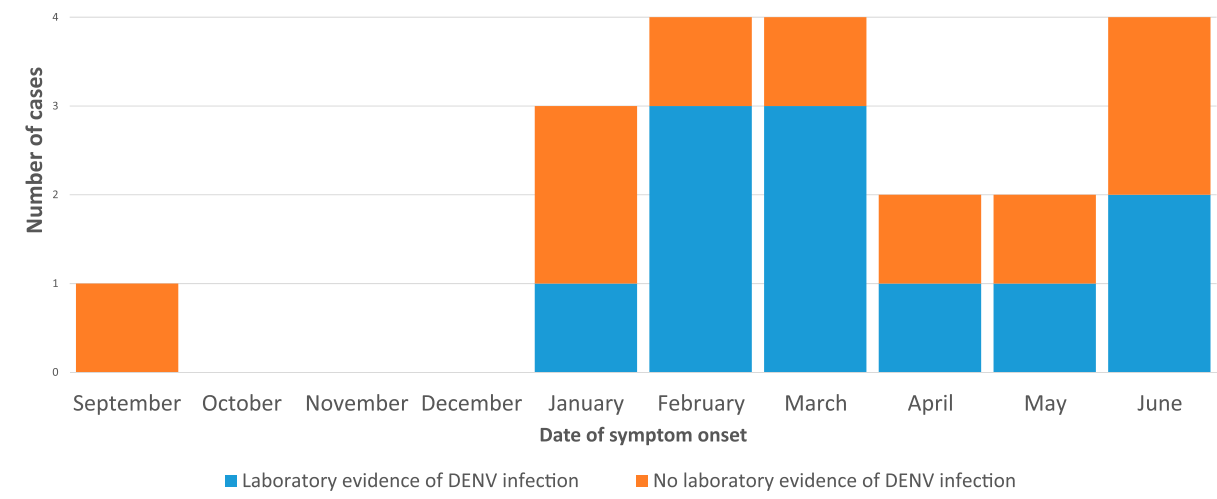

FIGURE 1. Laboratory evidence of dengue among Peace Corps volunteers in Timor-Leste who sought medical care for acute febrile illness or suspicion of dengue during September 2018 to June $2019(n=20)$. This figure appears in color at www.ajtmh.org. 


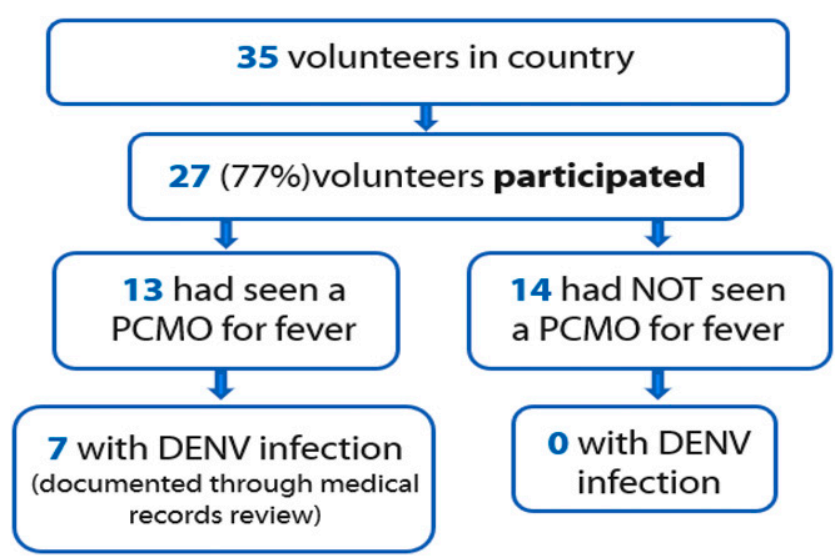

DENV = dengue virus $; \mathrm{PCMO}=$ Peace Corps medical officer

FIGURE 2. Overall participation of Peace Corps volunteers in a survey to identify factors associated with dengue virus infection, Timor-Leste, 2019. This figure appears in color at www.ajtmh.org.

cross reactivity. Anti-CHIKV IgM antibody was detected in another PCV; confirmatory testing by PRNT did not detect neutralizing anti-CHIKV antibodies.

For this analysis, participants with previously documented DENV infection through the medical records review and/or through diagnostic testing performed at the CDC Dengue Branch were included in the DENV infection group $(n=7)$ (Figure 3). No significant differences by DENV infection status were identified by demographic characteristics, vaccination history, municipality of residence, living situation, work type, work conditions, or nights spent outside the municipality of residence (Supplemental Table 1).

A total of $22(81 \%)$ participants reported at least one episode of fever during October 2018-June 2019, including all participants with evidence of DENV infection (Table 2). The only symptom significantly associated with DENV infection was myalgia $(P<0.05)$. Duration of illness was significantly longer among PCVs with evidence of DENV infection compared with those people without evidence of DENV infection (median: 10 versus 5 days; $P<0.05$ ). Among 22 PCVs who reported fever, 15 (68\%) were evaluated by their Peace Corps medical officer. Of these, seven $(47 \%)$ were diagnosed with dengue, all of whom had evidence of DENV infection. Of these, $5(71 \%)$ were hospitalized and required medical evacuation. The need for medical evacuation was determined by the attending physician; and given resources limitations in-country, patients who need hospitalization or presenting with worsening symptoms were evacuated.

All participants reported being bitten by mosquitoes during the investigation period, and most $(n=17,63 \%)$ reported being bitten most often during the evening (Table 3 ). Most participants reported being bitten most often at home $(n=14$, $56 \%$ ) and sleeping under a bed net every night ( $n=25,93 \%)$. No PCVs reported having used permethrin to treat clothes before or after arrival in Timor-Leste. Most PCVs $(n=16,59 \%)$ also reported having screens on the windows of their bedroom (Table 3), and roughly one-third $(n=8,30 \%)$ reported daily use of mosquito repellent. Most PCVs ( $n=24,89 \%)$ recalled completing training on mosquito avoidance, and 17 (63\%) and 15 (56\%) reported knowing that DENV was transmitted by mosquitoes and the risk of exposure to dengue in Timor-
Leste, respectively. No reported mosquito avoidance behaviors or knowledge were significantly associated with DENV infection.

\section{DISCUSSION}

The incidence of dengue among PCVs has been reported to follow the epidemic trends of regions and countries where PCVs work. ${ }^{12}$ Through the medical records review in this investigation, we observed that during a dengue epidemic in Timor-Leste, more than half of the PCVs in-country sought medical care for fever, and one-third had laboratory diagnostic evidence of dengue. Although none of the dengue patients met criteria for severe dengue, medical evacuation was required for two-thirds of PCVs with dengue, highlighting the importance of continued clinical training to identify and provide timely treatment of dengue patients in resource-limited settings. Although no specific mosquito avoidance behaviors were associated with DENV infection, all PCVs recalled being bitten by mosquitos and most reported being bitten most often at home, which emphasizes their continuous risk of for arboviral infections and the need to maintain and improve prevention measures.

By reviewing PCVs' medical records, we found the expected symptoms of dengue, including warning signs for progression to severe dengue. As expected, rash was significantly more common in volunteers with dengue. Rash has been estimated to occur in $50-82 \%$ patients with dengue, ${ }^{16-18}$ and its time of presentation and characteristics can be of use in identifying patients with dengue in areas where other acute febrile illnesses are endemic. ${ }^{19,20}$

Along with fever, all dengue patients identified through the medical record review presented with myalgia, and selfreported myalgia was significantly higher among participants in the survey and blood sample collection with evidence of dengue. Myalgia is commonly reported among dengue patients, more often in adults, and it has been more frequently reported in DENV-1 and DENV-3 infections. ${ }^{21,22}$

To increase awareness and improve knowledge of dengue clinical manifestations and management, re-training was provided to all Peace Corps medical officers providing medical care to PCVs around the world, and annual dengue clinical management refreshers were recommended along with the CDC online Dengue Clinical Case Management. ${ }^{23}$ As appropriate clinical management of dengue patients can significantly reduce mortality, ${ }^{24,25}$ improvement of clinical diagnosis and management was one component of the WHO Global

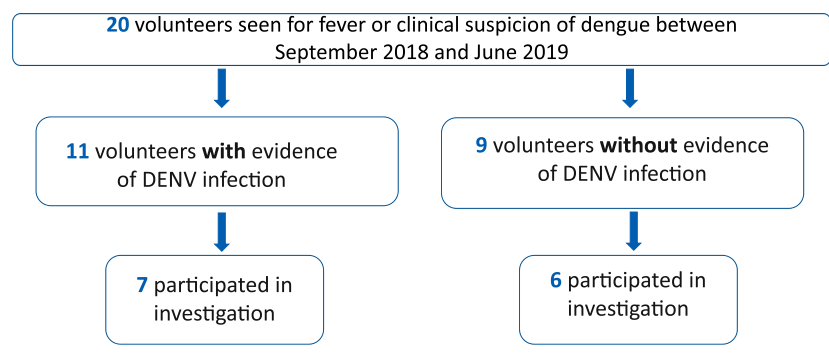

FIGURE 3. Participation of Peace Corps volunteers identified through the medical records review in a survey to identify factors associated with dengue virus infection, Timor-Leste, 2019. This figure appears in color at www.ajtmh.org. 
TABLE 2

Clinical characteristics of Peace Corps volunteers in Timor-Leste reporting illness episodes in October 2018-June 2019 by status of dengue virus infection $(n=27)$

\begin{tabular}{|c|c|c|c|c|}
\hline & $\begin{array}{l}\text { All participants, } \\
n=27(\%)\end{array}$ & $\begin{array}{l}\text { Participants with evidence of } \\
\text { DENV infection, } n=7(\%)\end{array}$ & $\begin{array}{l}\text { Participants without evidence } \\
\text { of DENV infection, } n=20 \text { (\%) }\end{array}$ & $P$ value ${ }^{*}$ \\
\hline \multicolumn{5}{|l|}{ Demographic characteristics } \\
\hline Age in years, median (range) & $25(22-67)$ & $26(23-45)$ & $25(22-67)$ & 0.4210 \\
\hline Female gender, $n(\%)$ & $10(37)$ & $4(57)$ & $6(30)$ & 0.3648 \\
\hline Acute febrile illness & $22(81)$ & 7 (100) & $15(75)$ & 0.2834 \\
\hline Nausea or vomiting $\dagger$ & $9(41)$ & $3(43)$ & $6(40)$ & 1.0000 \\
\hline Rash† & $4(18)$ & $3(43)$ & $1(7)$ & 0.0766 \\
\hline Headache† & $21(95)$ & $7(100)$ & $14(93)$ & 1.0000 \\
\hline Eye pain $†$ & $4(18)$ & $2(29)$ & $2(13)$ & 0.5646 \\
\hline Arthralgia† & $3(14)$ & $2(29)$ & $1(7)$ & 0.2273 \\
\hline Myalgia† & $9(41)$ & $6(86)$ & $3(20)$ & 0.0066 \\
\hline Abdominal pain or tenderness $†$ & $5(23)$ & $1(14)$ & $4(27)$ & 1.0000 \\
\hline Diarrhea† & $10(45)$ & $2(29)$ & $8(53)$ & 0.3808 \\
\hline Runny nose & $7(32)$ & $2(29)$ & $5(33)$ & 1.0000 \\
\hline Sore throat $\dagger$ & $8(36)$ & $2(29)$ & $6(40)$ & 1.0000 \\
\hline Red eyest & $1(5)$ & $1(14)$ & $0(0)$ & 0.3182 \\
\hline Calf pain $†$ & $1(5)$ & $1(14)$ & $0(0)$ & 0.3182 \\
\hline Red or swollen joints $t$ & $1(5)$ & $1(14)$ & $0(0)$ & 0.3182 \\
\hline $\begin{array}{l}\text { Illness duration in days, median† } \\
\text { (range) }\end{array}$ & $5(2-30)$ & $10(8-17)$ & $5(2-30)$ & 0.0007 \\
\hline
\end{tabular}

$P<0.05$ = statistically significant.

†Denominator excludes individuals who did not report an acute febrile illness $(n=5)$. All calculations are based on participants who reported an acute febrile illness $(n=22)$ with evidence of DENV infection $(n=7)$ and without evidence of DENV infection $(n=15)$.

Strategy for Dengue Prevention and Control to reduce dengue case-fatality rate by at least $50 \%$ by $2020 .{ }^{26}$ Additionally, maintenance of a list of medical evacuation criteria (e.g., warning signs for severe dengue), with periodic review to ensure alignment with local resources and current guidelines, was recommended for all Peace Corps posts where dengue is endemic. This is especially important in posts with similar conditions to Timor-Leste, where there is an important limitation in immediate hospital services for PCVs.

Because all PCVs recalled mosquito bites during their time in Timor-Leste, continued implementation of mosquito bite prevention measures should be a priority to protect PCVs against dengue and other arboviruses in this population. Volunteers reported that they were most frequently bitten by mosquitoes at home, and 11 (41\%) reported not having screens on their bedroom windows. In some settings, window screens have been associated with reduced infestations of Aedes aegypti mosquitoes and potential reduction in risk of DENV infection. ${ }^{27-29}$ CDC recommends that travelers to and residents of the tropics reside in homes with intact window screens to avoid DENV infection. ${ }^{30}$ The use of bed nets has been associated with protection from DENV infection in some studies. $^{31-33}$ Aedes mosquitoes are more active during the day, but the range of activity can include early hours of the morning and evening when bed nets can help prevent Aedes mosquito bites. CDC recommends the use of a bed net for dengue prevention, and PCVs should be encouraged to continue this practice.

CDC also recommends frequent use of mosquito repellents to avoid mosquito bites and DENV infection, and reapplication according to label instructions. ${ }^{30}$ As only one-third of interviewed PCVs reported daily use of repellent, this demonstrates an opportunity to improve prevention measures among PCVs in Timor-Leste. The provision and use of permethrin to apply on clothes and other articles prior to PCV's departure and on a regular basis while in-country could also improve protection of PCVs against mosquito bites. On completion of this investigation, the Peace Corps ensured that posts could provide permethrin spray for clothes.

Finally, CDC also recommends, when possible, residing in homes with air conditioning to avoid mosquito bites, as homes with air conditioning are typically well sealed and consequently are less accessible to mosquitos. ${ }^{34}$ Access to air conditioning for most PCVs is limited because of living conditions. Air conditioning use was only reported by one PCV in Timor-Leste; therefore, we were not able to assess its potential protection against DENV infection in this investigation nor the feasibility of improving access to air conditioning for PCVs in Timor-Leste. We did not assess other mosquito control measures taken by PCVs, such as frequent removal of standing water in domestic and peri-domestic environments. Peace Corps medical officers provided information on prevention practices during the outbreak, including mosquito breeding sites reduction; but the feasibility or successful implementation of this approach is unknown, because keeping water in open containers was reported as a common practice in Timor-Leste by PCVs.

The findings of this investigation are subject to some limitations. First, as the total participants in the survey was 27 out of the $35 \mathrm{PCVs}$ in-country and only 11 dengue patients were identified, this investigation's power to detect factors associated with DENV infection was limited. Second, as DENV transmission in Timor-Leste was ongoing for at least 6 months before the survey and blood sample collection was conducted, we cannot rule out seroreversion and consequent misclassification of some participants. The duration of detectability of anti-DENV IgM antibody is not well defined, including how it may differ by infecting DENV serotype, status of primary versus secondary DENV infection, and symptomatic versus asymptomatic infection. ${ }^{35}$ Also, given the period for which participants were asked questions regarding symptoms and preventive behavior, recall bias may be a limitation for the accuracy and completeness of the 
TABLE 3

Mosquito bite prevention measures and mosquito bites-related characteristics reported by Peace Corps volunteers in Timor-Leste by status of dengue virus infection, October 2018-June $2019(n=27)$

\begin{tabular}{|c|c|c|c|c|}
\hline Prevention measure/Characteristic & $\begin{array}{c}\text { All } \\
\text { participants, } \\
n=27(\%)\end{array}$ & $\begin{array}{l}\text { Participants with } \\
\text { evidence of DENV } \\
\text { infection, } n=7(\%)\end{array}$ & $\begin{array}{l}\text { Participants without } \\
\text { evidence of DENV } \\
\text { infection, } n=20(\%)\end{array}$ & $P$ value $^{*}$ \\
\hline Recalls mosquito bites & $27(100)$ & $7(100)$ & $20(100)$ & - \\
\hline When most often bitten by mosquitoes $\uparrow \S$ & & & & 0.0520 \\
\hline Morning & $1(4)$ & $0(0)$ & $1(6)$ & \\
\hline Afternoon & $2(8)$ & $2(33)$ & $0(0)$ & \\
\hline Early evening & $7(29)$ & $1(17)$ & $6(33)$ & \\
\hline Late evening & $20(42)$ & $1(17)$ & $9(50)$ & \\
\hline Nighttime & $3(13)$ & 2 (33) & $1(6)$ & \\
\hline Don't recall & $1(4)$ & $0(0)$ & $1(6)$ & \\
\hline \multicolumn{5}{|l|}{ Screens and $A C$ use } \\
\hline Screens on doors in bedroom & $2(7)$ & $1(14)$ & $1(5)$ & 0.4587 \\
\hline Screens on doors in home & $1(4)$ & $1(14)$ & $0(0)$ & 0.2593 \\
\hline Screens on windows in bedroom & $16(59)$ & $5(71)$ & $11(55)$ & 0.6618 \\
\hline Screens on windows in home $§$ & $3(11)$ & $1(14)$ & $2(10)$ & 1.0000 \\
\hline AC use in bedroom & $1(4)$ & $1(14)$ & $0(0)$ & 0.2593 \\
\hline AC use in home†§ & $1(4)$ & $1(14)$ & $0(0)$ & 0.2692 \\
\hline Repellent use & & & & 0.2921 \\
\hline At least once a day & $8(30)$ & $1(14)$ & $7(35)$ & \\
\hline Weekly or less often & $4(15)$ & $0(0)$ & $4(20)$ & \\
\hline Whenever notices mosquitoes & $13(48)$ & $6(86)$ & $7(35)$ & \\
\hline Never & $1(4)$ & $0(0)$ & $1(5)$ & \\
\hline Don't recall & $1(4)$ & $0(0)$ & $1(5)$ & \\
\hline Bed net use & & & & 1.0000 \\
\hline Every night & $25(93)$ & $7(100)$ & $18(90)$ & \\
\hline Less than monthly & $1(4)$ & $0(0)$ & $1(5)$ & \\
\hline Never & $1(4)$ & $0(0)$ & $1(5)$ & \\
\hline Permethrin use on clothes before arriving $\ddagger \S$ & $0(0)$ & $0(0)$ & $0(0)$ & - \\
\hline Permethrin use on clothes since arriving†‡ & $0(0)$ & $0(0)$ & $0(0)$ & - \\
\hline Wears long sleeve shirts & $12(44)$ & $1(14)$ & $11(55)$ & 0.0914 \\
\hline Wears long pants & $15(56)$ & $3(43)$ & $12(60)$ & 0.6618 \\
\hline Wears a hat & $11(41)$ & $2(29)$ & $9(45)$ & 0.6618 \\
\hline Uses mosquito coils & $2(7)$ & $1(14)$ & $1(5)$ & 0.4587 \\
\hline Uses insecticide aerosols & $8(30)$ & $2(29)$ & $6(30)$ & 1.0000 \\
\hline Where most often bitten by mosquitoes $\dagger \ddagger \S$ & & & & 0.1650 \\
\hline At home & $14(56)$ & $3(50)$ & $11(58)$ & \\
\hline While shopping/running errands & $1(4)$ & $1(17)$ & $0(0)$ & \\
\hline During leisure activities & $5(20)$ & $2(33)$ & $3(16)$ & \\
\hline Completed training on mosquito avoidance§ & $24(89)$ & $7(100)$ & $17(85)$ & 0.5453 \\
\hline Knew dengue transmitted by mosquitoes§ & $17(63)$ & $3(43)$ & $14(70)$ & 0.3648 \\
\hline Knew exposure to dengue in TL§ & $15(56)$ & $4(57)$ & $1155)$ & 1.0000 \\
\hline
\end{tabular}

collected data, especially for detailed information on frequency of using prevention methods. To try to avoid bias in the participants responses toward the socially desirable behaviors, identifiable data from questionnaires was kept confidential, reviewed, and analyzed by CDC staff and not shared with Peace Corps leadership, and this was communicated to all participants before and during the questionnaire completion. As some desirable preventive behaviors like daily use of repellent were reported in less than half of PCVs in both groups, we do not expect the results of the questionnaire to be biased. Finally, although the recommendations regarding behavioral approaches to reduce exposure to mosquitos may be of use for PCVs in other areas of the tropics, and for other long-term care travelers, the specific findings of this investigation may not be generalizable to other PCVs or other long-term care travelers, because the specific characteristics of each group of travelers and each destination vary widely.

As the global burden of dengue has increased in recent years, ${ }^{36,37}$ so has the incidence of dengue among travelers to the tropics. ${ }^{38}$ This burden is expected to continue to grow in Asia, Sub-Saharan Africa, and Latin America, with global estimates of more than 50 million febrile illness cases and 2 million hospitalizations per year, ${ }^{39}$ further increasing the risk for travelers to these areas. Dengue infection risk in longterm travelers (more than 6 months) and business expatriates (those who reside in another country for occupational purposes, and will return to their country of origin after their assignment is completed) has been associated with increased duration of assignments and local epidemiology of dengue in the destination. ${ }^{40,41}$ As long-term travelers to dengue endemic areas with potential occurrence of outbreaks during their 
assignment of $>2$ years, $\mathrm{PCV}$ s will continue to be at increased risk for dengue and other arboviral diseases.

Different than many other long-term travelers, PCVs undergo a complete medical assessment before their assignment and receive standardized training on prevention measures for different risks and conditions. Because most volunteers recalled completing training on mosquito avoidance before their departure, the inclusion of specific information on dengue and other arboviral diseases (chikungunya, Zika) in the training schedule may be beneficial. This approach of including relevant information on dengue risk factors could also be helpful for pre-travel counseling for other types of long-term travelers. An annual refresher on mosquito avoidance behavior and arboviral diseases is recommended for PCVs, along with refreshers when outbreaks are declared, and they should include the most common clinical presentation for dengue and key symptoms like fever, rash, and myalgia, as found in this report. The use of peer accounts and personal experiences from PCVs with dengue may provide a more impactful and lasting notion of the real effects and potential complications of dengue among incoming PCVs. Besides pre-travel training and annual refreshers, several mechanisms have been identified and have been used to communicate to PCVs or to host families crucial information on dengue outbreaks and prevention measures, including the use of newsletters, messages through group chats, discussions during medical care sought for other reasons, and inclusion of dengue prevention information during host families' orientation.

DENV infection risk is determined by multiple factors, including socioeconomic, behavioral, and environmental factors. There is limited information available regarding the association between the nature of the work in volunteers and other aid workers and an increased risk of DENV infection, ${ }^{40}$ but some studies have found an increased risk of dengue with specific activities that might increase the risk for mosquito bites, like playing outdoors sports. ${ }^{42}$ Our investigation did not find any differences in DENV infection by type of work (education versus community economic development), work conditions (indoors versus outdoors), or mosquito avoidance behaviors. However, given the limitations of this investigation that include a small sample size, preventive behaviors should continue to be encouraged to decrease risk of DENV infection among PCVs. The results of this investigation and subsequent recommendations may also be of use to PCVs in other countries where dengue is endemic.

Received January 7, 2021. Accepted for publication March 8, 2021.

Published online April 26, 2021.

Note: Supplemental table appears at www.ajtmh.org

Acknowledgments: We thank Kavita Pullapilly, Deolindo Gusmao, and Erica Franz (Timor Leste Office, Peace Corps) for their support with the logistics necessary to collect data and blood samples in Timor-Leste. We also thank Andre Robinson and Ken Puyak (Post Logistics and Support Division, Peace Corps Headquarters), Rafael Tosado, and Nicole Medina (CDC Dengue Branch, San Juan, Puerto Rico) for their role in the successful sample transportation from Timor-Leste to San Juan, Puerto Rico. Special thanks to Madeline Wilks (Office of Medical Services, Peace Corps Headquarters) for her valuable input during the design and implementation of the activities described in this manuscript. We also thank the Peace Corps Regional Medical Officers in Thailand, Alexander Gonta and Mustafa Sulemanji, for their assistance and support in conducting this outbreak investigation.
Financial support: The investigation was supported, but not specifically funded, by CDC and the U.S. Peace Corps.

Disclaimer: The views expressed in this article are ours and do not necessarily represent the official position of the U.S. Centers for Disease Control and Prevention or the U.S. Public Health Service.

Authors' addresses: Liliana Sánchez-González, Chelsea G. Major, Jorge L. Munoz-Jordan, Freddy A. Medina, Gabriela Paz-Bailey, and Tyler M. Sharp, Centers for Disease Control and Prevention, Dengue Branch, San Juan, Puerto Rico, E-mails: naq5@cdc.gov, Ihi5@ cdc.gov, ckq2@cdc.gov, fkt3@cdc.gov, gmb5@cdc.gov, and iyp4@ cdc.gov. Margaret Venuto, Scott Poe, and Karen Becker, Office of Health Services, Epidemiology and Surveillance Unit, Paul Coverdell Peace Corps HQ/Office of Health Services, Washington, DC, E-mails: mvenuto@peacecorps.gov, spoe@peacecorps.gov, and kbecker2@ peacecorps.gov. Leonardus Baskara and Sevinj Abdiyeva, Office of Health Services, Timor-Leste Country Office, Paul Coverdell Peace Corps HQ/Office of Health Services, Washington, DC, E-mails: lbaskara@peacecorps.gov and sabdiyeva@peacecorps.gov. Daniel Murphy, Office of AIDS Research, Office of the Director, National Institutes of Health, Rockville, MD, E-mail: danny.murphy@nih.gov. Kyle Petersen, Department of Preventive Medicine \& Biostatistics, Uniformed Services University of the Health Sciences, Bethesda, MD, E-mail: kyle.petersen@usuhs.edu.

This is an open-access article distributed under the terms of the Creative Commons Attribution (CC-BY) License, which permits unrestricted use, distribution, and reproduction in any medium, provided the original author and source are credited.

\section{REFERENCES}

1. World Health Organization, 2009. Dengue Guidelines for Diagnosis, Treatment, Prevention and Control. Geneva, Switzerland: WHO.

2. Clapham HE, Cummings DAT, Johansson MA, 2017. Immune status alters the probability of apparent illness due to dengue virus infection: evidence from a pooled analysis across multiple cohort and cluster studies. PLoS Negl Trop Dis 11: e0005926.

3. Wilder-Smith A, Ooi EE, Horstick O, Wills B, 2019. Dengue. Lancet 393: 350-363.

4. Pan American Health Organization, 2016. Dengue: Guidelines for Patient Care in the Region of the Americas, 2nd ed. Washington, DC: Pan American Sanitary Bureau, Regional Office of the World Health Organization.

5. Leo YS, Gan VC, Ng EL, Hao Y, Ng LC, Pok KY, Dimatatac F, Go CJ, Lye DC, 2013. Utility of warning signs in guiding admission and predicting severe disease in adult dengue. BMC Infect Dis 13: 498.

6. Lam PK et al., 2013. Clinical characteristics of dengue shock syndrome in Vietnamese children: a 10-year prospective study in a single hospital. Clin Infect Dis 57: 1577-1586.

7. Wangdi K, Clements ACA, Du T, Nery SV, 2018. Spatial and temporal patterns of dengue infections in Timor-Leste, 2005-2013. Parasit Vectors 11: 9.

8. Anderson EM, Davis JA, 2014. Field evaluation of the response of Aedes albopictus (Stegomyia albopicta) to three oviposition attractants and different ovitrap placements using black and clear autocidal ovitraps in a rural area of Same, Timor-Leste. Med Vet Entomol 28: 372-383.

9. Kalayanarooj S, Rimal HS, Andjaparidze A, Vatcharasaevee V, Nisalak $A$, Jarman RG, Chinnawirotpisan P, Mammen MP, Holmes EC, Gibbons RV, 2007. Clinical intervention and molecular characteristics of a dengue hemorrhagic fever outbreak in Timor Leste, 2005. Am J Trop Med Hyg 77: 534-537.

10. Deen J, Matos Lda C, Temple B, Su JY, da Silva J, Liberato S, da Silva V, Soares Al, Joshi V, Moon S, Tulloch J, Martins J, Mulholland K, 2013. Identifying national health research priorities in Timor-Leste through a scoping review of existing health data. Health Res Policy Syst 11: 8.

11. IFRC Information Bulletin, April 29, 2019. Timor-Leste: Dengue Outbreak. Geneva, Switzerland: International Federation of Red Cross and Red Crescent Societies.

12. Ferguson RW, Henderson SJ, Lee EA, Jung P, 2016. Dengue in Peace Corps volunteers, 2000-14. J Travel Med 23. 
13. DENV Detect ${ }^{T M} \operatorname{IgM}$ Capture ELISA. Seattle, WA: InBios International, Inc.

14. Lanciotti RS, Kosoy OL, Laven JJ, Velez JO, Lambert AJ, Johnson AJ, Stanfield SM, Duffy MR, 2008. Genetic and serologic properties of Zika virus associated with an epidemic, Yap State, Micronesia, 2007. Emerg Infect Dis 14: 1232-1239.

15. Martin DA, Muth DA, Brown T, Johnson AJ, Karabatsos N, Roehrig JT, 2000. Standardization of immunoglobulin M capture enzyme-linked immunosorbent assays for routine diagnosis of arboviral infections. J Clin Microbiol 38: 1823-1826.

16. Thomas EA, John M, Kanish B, 2010. Mucocutaneous manifestations of dengue fever. Indian J Dermatol 55: 79-85.

17. Waterman SH, Gubler DJ, 1989. Dengue fever. Clin Dermatol 7: 117-122.

18. Itoda I et al., 2006. Clinical features of 62 imported cases of dengue fever in Japan. Am J Trop Med Hyg 75: 470-474.

19. Gregory CJ, Santiago LM, Arguello DF, Hunsperger E, Tomashek $\mathrm{KM}, 2010$. Clinical and laboratory features that differentiate dengue from other febrile illnesses in an endemic area-Puerto Rico, 2007-2008. Am J Trop Med Hyg 82: 922-929.

20. Quinn EJ, Cheong AH, Calvert JK, Higgins G, Hahesy T, Gordon $\mathrm{DL}, \mathrm{Carr} \mathrm{JM}, 2018$. Clinical features and laboratory findings of travelers returning to South Australia with dengue virus infection. Trop Med Infect Dis 3: 6.

21. Garg RK, Malhotra HS, Jain A, Malhotra KP, 2015. Dengueassociated neuromuscular complications. Neurol India 63: 497-516.

22. Lovera D, Martinez-Cuellar C, Galeano F, Amarilla S, Vazquez C, Arbo A, 2019. Clinical manifestations of primary and secondary dengue in Paraguay and its relation to virus serotype. $J$ Infect Dev Ctries 13: 1127-1134.

23. CDC, 2020. Dengue Clinical Case Management (DCCM) Course. Atlanta, GA: Centers for Disease Control and Prevention. Available at: https://www.cdc.gov/dengue/training/cme/ccm/ index.html. Accessed October 26, 2020.

24. Thomas L, Moravie V, Besnier F, Valentino R, Kaidomar S, Coquet LV, Najioullah F, Lengelle F, Cesaire R, Cabie A, Working Group on D, 2012. Clinical presentation of dengue among patients admitted to the adult emergency department of a tertiary care hospital in Martinique: implications for triage, management, and reporting. Ann Emerg Med 59: 42-50.

25. Marra AR, de Matos GF, Janeri RD, Machado PS, Schvartsman C, Dos Santos OF, 2011. Managing patients with dengue fever during an epidemic: the importance of a hydration tent and of a multidisciplinary approach. BMC Res Notes 4: 335.

26. World Health Organization, 2012. Global Strategy for Dengue Prevention and Control 2012-2020. Available at: https:// apps.who.int/iris/handle/10665/75303. Accessed April 5, 2021

27. Ko YC, Chen MJ, Yeh SM, 1992. The predisposing and protective factors against dengue virus transmission by mosquito vector. Am J Epidemiol 136: 214-220.

28. Igarashi A, 1997. Impact of dengue virus infection and its control. FEMS Immunol Med Microbiol 18: 291-300.
29. Manrique-Saide P et al., 2015. Use of insecticide-treated house screens to reduce infestations of dengue virus vectors, Mexico. Emerg Infect Dis 21: 308-311.

30. CDC, 2019. Dengue-Prevent Mosquito Bites. Atlanta, GA: Centers for Disease Control and Prevention, National Center for Emerging and Zoonotic Infectious Diseases (NCEZID), Division of Vector-Borne Diseases (DVBD). Available at: https:// www.cdc.gov/dengue/prevention/prevent-mosquito-bites.html\#: :text=Use\%20air\%20conditioning\%2C\%20if\%20available, \% 2C\%20flowerpots\%2C\%20or\%20trash\%20containers. Accessed April 5, 2021.

31. Ferede G, Tiruneh M, Abate E, Wondimeneh Y, Damtie D, Gadisa E, Howe R, Aseffa A, Tessema B, 2018. A serologic study of dengue in northwest Ethiopia: suggesting preventive and control measures. PLoS Negl Trop Dis 12: e0006430.

32. Kenneson A, Beltran-Ayala E, Borbor-Cordova MJ, Polhemus ME, Ryan SJ, Endy TP, Stewart-Ibarra AM, 2017. Socialecological factors and preventive actions decrease the risk of dengue infection at the household-level: results from a prospective dengue surveillance study in Machala, Ecuador. PLoS Negl Trop Dis 11: e0006150.

33. Sharp TM et al., 2015. Underrecognition of dengue during 2013 epidemic in Luanda, Angola. Emerg Infect Dis 21: 1311-1316.

34. Reiter $P$ et al., 2003. Texas lifestyle limits transmission of dengue virus. Emerg Infect Dis 9: 86-89.

35. Sharp TM, Fischer M, Munoz-Jordan JL, Paz-Bailey G, Staples JE, Gregory CJ, Waterman SH, 2019. Dengue and Zika virus diagnostic testing for patients with a clinically compatible illness and risk for infection with both viruses. MMWR Recomm Rep 68: 1-10.

36. Shepard DS, Undurraga EA, Halasa YA, Stanaway JD, 2016. The global economic burden of dengue: a systematic analysis. Lancet Infect Dis 16: 935-941.

37. Stanaway JD et al., 2016. The global burden of dengue: an analysis from the Global Burden of Disease Study 2013. Lancet Infect Dis 16: 712-723.

38. Wilder-Smith A, 2018. Risk of dengue in travelers: implications for dengue vaccination. Curr Infect Dis Rep 20: 50.

39. Cattarino L, Rodriguez-Barraquer I, Imai N, Cummings DAT, Ferguson NM, 2020. Mapping global variation in dengue transmission intensity. Sci Transl Med 12: eaax4144.

40. Visser JT, Edwards CA, 2013. Dengue fever, tuberculosis, human immunodeficiency virus, and hepatitis $C$ virus conversion in a group of long-term development aid workers. J Travel Med 20: 361-367.

41. Lim PL, Han P, Chen LH, MacDonald S, Pandey P, Hale D, Schlagenhauf $P$, Loutan L, Wilder-Smith A, Davis XM, Freedman DO, GeoSentinel Surveillance N, 2012. Expatriates ill after travel: results from the Geosentinel Surveillance Network. BMC Infect Dis 12: 386.

42. Liu J, Tian X, Deng Y, Du Z, Liang T, Hao Y, Zhang D, 2019. Risk factors associated with dengue virus infection in Guangdong Province: a community-based case-control study. Int J Environ Res Public Health 16: 617. 